\title{
A REAL-TIME FACE RECOGNITION SYSTEM USING MULTIPLE MEAN FACES AND DUAL MODE FISHERFACES
}

\author{
Jongmoo Choi, Sanghoon Lee, Chilgee Lee, Juneho Yi \\ School of Electrical and Computer Engineering \\ Sungkyunkwan University \\ 300, ChunChundong, Jangan-gu, Suwon 440-746, Korea \\ jjmchoi, armofgod, cslee, jhyi\}@ece.skku.ac.kr
}

\begin{abstract}

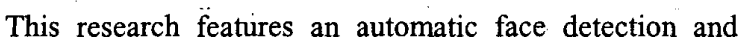
recognition system. The purpose of the system is for access control to a building or an office. The main feature of the system is face detection and face recognition robust to illumination changes. A novel template matching technique using multiple mean faces (MMF) of various sizes, luminance, and rotations is employed to achieve robust face detection. The system is also capable of operating in two different modes for face recognition: under normal illumination condition and under severe illumination changes.
\end{abstract}

\section{INTRODUCTION}

Security systems based on automatic face recognition have advantages over systems using other biometric identification techniques in that face recognition provides a more natural means of person identification. However, face detection and recognition in lighting variations is a very hard problem [1]. We have developed a practical working system based on face detection and recognition for the purpose of application to access control to a building or an office.

The main features of the system are as follows. First, we have achieved face detection robust to illumination changes. We have developed a novel template matching technique for face detection using multiple mean faces (MMF) of various sizes, luminance, and rotations. The technique combined with motion detection and skin color segmentation for fast face detection. We estimate brightness of skin color region in the input image and then apply appropriate templates of similar brightness to detect not only the facial region but also its size and rotation. This template matching is also computationally efficient because actual computation is confined to the region of interest (ROI) obtained from motion and skin color segmentation. Second, for face recognition, we have enhanced the recognition performance by enabling the system to not only work under normal lighting condition but also operate in a particularly tuned way when there are severe illumination changes.

In the following section, we give a brief overview of the entire system. Section 3 and 4 describe face detection and face recognition parts of the system respectively. We report the experimental results on the recognition performance of the system in section 5 .

\section{SYSTEM OVERVIEW}

The system can operate with a normal PC camera of 300,000 pixel resolution and the illumination condition for normal operation is interior lighting brighter than 60 lux. It takes less than 0.5 second to process an entire computation cycle from image capture to face recognition. The normal operating range from camera to a person to be identified is within $3 \mathrm{~m}$. If the distance from camera to a person is more than $3 \mathrm{~m}$, the system only tries to detect the person's face. The performance of the system is robust to illumination changes and eye glasses. Face rotation about 30 degrees in the image plane and partial occlusion of a face are also allowed. Fig. 1 shows a block diagram of the entire system.

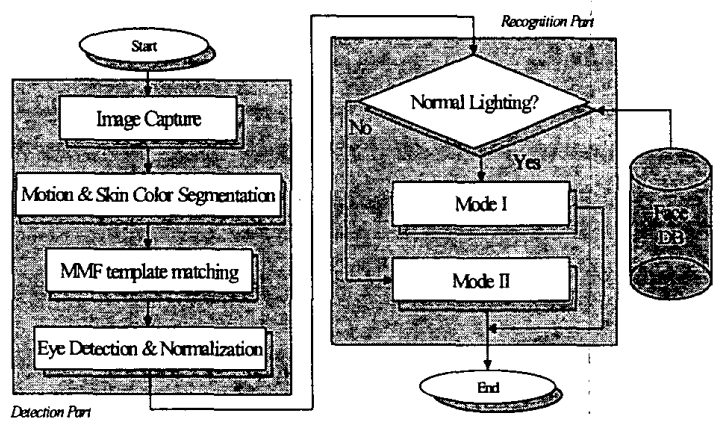

Fig. 1 System overview 


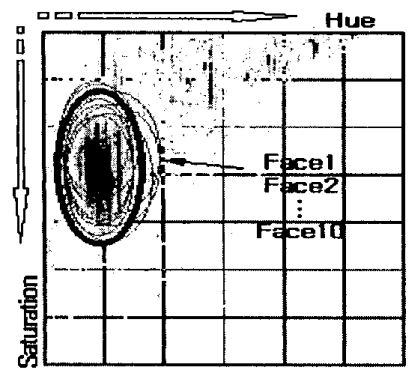

Fig. 2 Skin color distribution

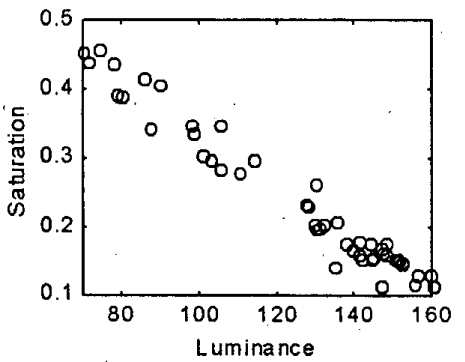

Fig. 3 Luminance vs. Saturation of skin color

The system is divided into face detection and face recognition parts. The detection part consists of (1) motion and skin color segmentation, (2) MMF based template matching and (3) eye detection and normalization of detected face regions based on the eyes location. The normalization refers to rotation, scaling and alignment of detected face regions to be input to the recognition part. For the recognition of detected faces, we have employed SKKUfaces method [7] that is an enhanced Fisherfaces method. The SKKUfaces method has two operating modes in order to tweaking the recognition performance: one is for normal illumination condition and the other for severe illumination changes.

\section{FACE DETECTION}

The first step is to select candidate face regions by motion and skin color segmentation. The second step is to locate face position by MMF based template matching. Finally, face regions are normalized by using the eyes locations.

\subsection{Motion and Skin Color Segmentation Robust to Illumination Changes}

The HSI color model is commonly used in color image segmentation because it is relatively easy to separately consider the luminance and the chrominance components [2]. However, it is well known that the luminance affects

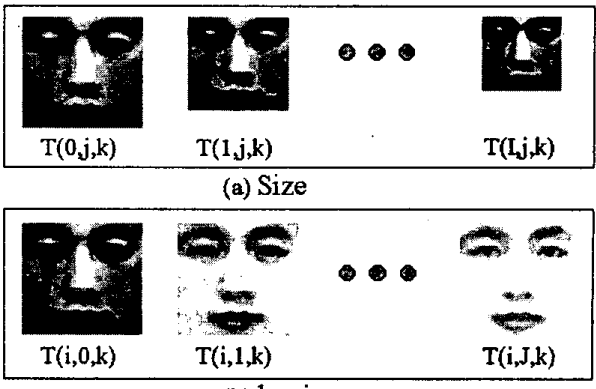

(b) luminance

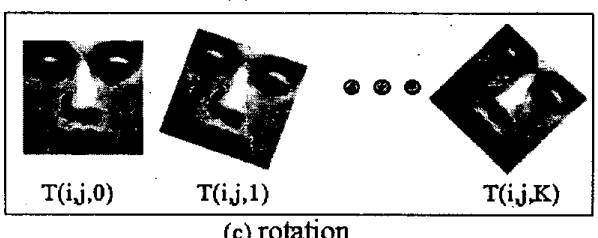

Fig. 4 multiple mean face (MMF) templates

the saturation. Fig. 2 shows the cumulative distribution of HS values of ten faces from the same ethnic group. They form a relatively tight cluster in the HS color space that can be approximately modeled by an ellipse. As the illumination varies, the elliptical distribution also changes. We have observed the relation between luminance and saturation as shown in Fig. 3. Based on this observation, the system is implemented to adaptively set the threshold values for skin color segmentation when the luminance value changes. However, in the case that changes of image brightness are severe, skin color region is not segmented correctly. To circumvent this problem we have also used motion segmentation. We find skin color region in the region obtained from motion segmentation.

\subsection{Detection of Face Regions Using MMF Based Template Matching}

The regions obtained from motion and skin color segmentation may contain multiple faces, hands or other regions similar to skin color. We select only face regions using the MMF based template matching technique. The previous method using a mean face image to detect face [10] is not well applied in the case of luminance changes. In our method, we generate multiple mean face images that consider various sizes, rotations and luminance changes as shown in Fig. 4. We estimate the brightness of skin color region in the input image in order to locate the face position. We first use upright MMF templates with similar brightness. The face position is determined by finding the location in the image with the maximum correlation value. Finally, to estimate the angle of face rotation, we use template matching with rotated MMF templates (Fig. 4 (c)). 


\subsection{Eye Detection and Normalization of a Face Region}

Eye detection is crucial in our system to judge a candidate face region as a real face region. As shown in Fig. 5 (a), a moving window searches for a black region, which corresponds to the pupil of the eye. The search location directs from down to top in the trajectory of an ellipse. We have learned from many trials that this way, the window locations do not go into the regions of hair or eyebrows when the face is rotated. As can be seen in Fig. 5 (b) - (d), eyes are correctly detected and located. The normalization step is composed of linear transformation, histogram equalization and masking. Using the location of detected eyes, the linear transformation rotates and scales the face to the size of $50 \times 50$ image. The histogram equalization is employed to make the facial image have good contrast. Lastly, the hair style and the background are removed as much as possible to only obtain the pure facial part. We call this 'masking' process.

\section{FACE RECOGNITION USING DUAL MODE FISHERFACES}

For the recognition of faces, we have employed the SKKUfaces method :[7] illustrated in Fig. 6. The SKKUfaces method is similar to the Fisherfaces method in that it first performs PCA (principal component analysis) and apply LDA (linear discriminant analysis) to the eigenrepresentation computed by PCA. It is well known that the

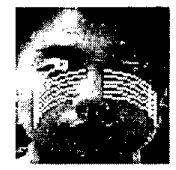

(a)

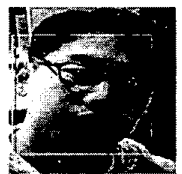

(b)

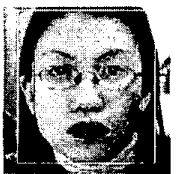

(c)

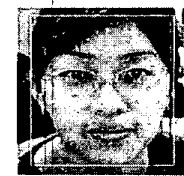

(d)
PCA step is a requirement in Fisherfaces-like approaches to guarantee that between-class scatter matrix be invertible. The SKKUfaces method is different from the Fisherfaces method in that it applies LDA to the eigen-representation computed by PCA with first few eigenvectors dropped. The effect is that face variations due to severe illumination changes are effectively removed but important information for the recognition of faces under normal illumination condition may be lost as well. For this reason, in this work, let us call the SKKUfaces the dual mode of the Fisherfaces. Another advantage of SKKUfaces is the efficient computation of the between-class scatter and within-class scatter matrices [7]. Our system works in two different modes. One is the Fishefaces mode that is supposed to be used for the recognition of faces under normal illumination condition. The other is the SKKUfaces mode for face recognition under severe illumination changes. In the SKKUfaces mode, only the top eigenvector is eliminated in the current implementation of the system. The system detects normal lighting condition basically by checking the symmetry of brightness pattern of a facial imàge.

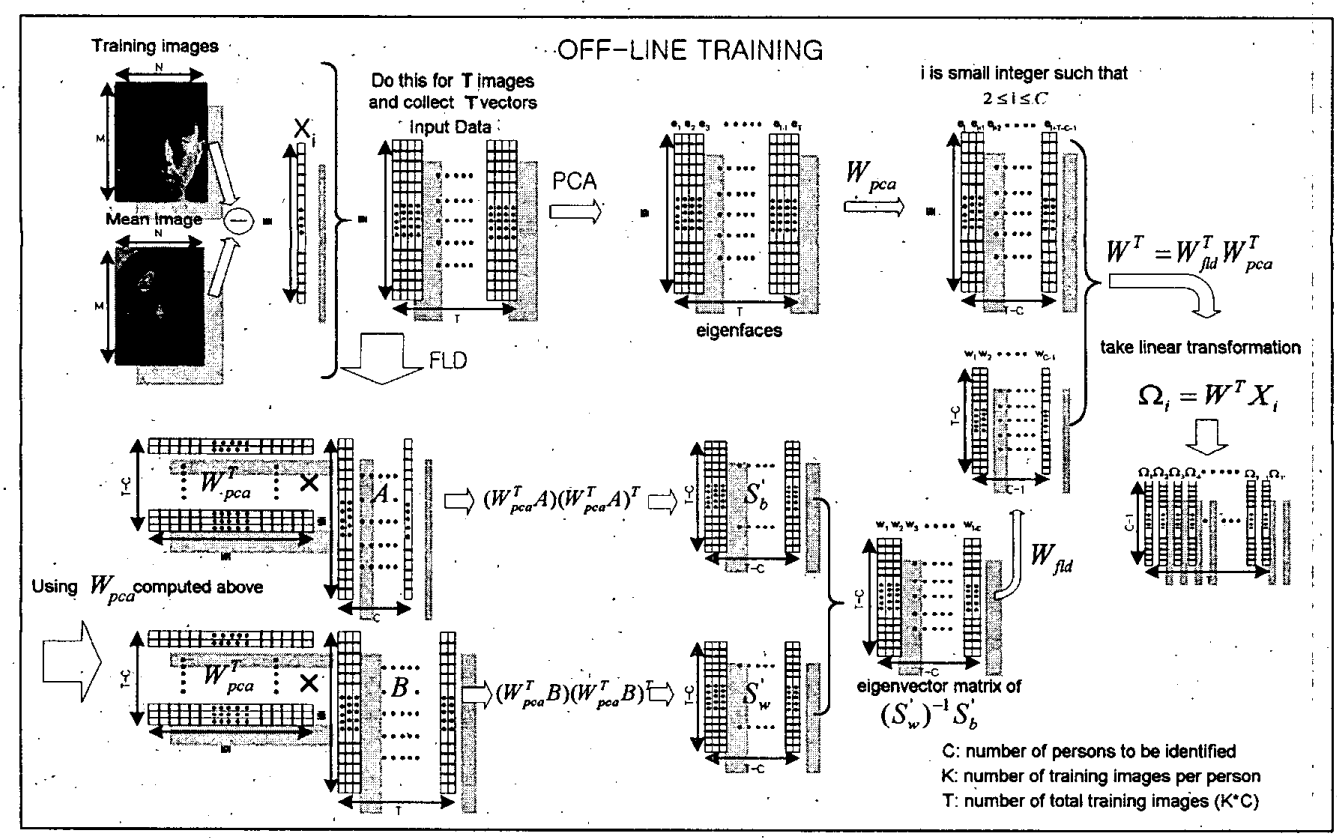

Fig. 6 The training phase of the Fisherfaces $(i=1)$ and the dual mode of the Fisherfaces $(i \geq 2)$ 


\section{EXPERIMENTAL RESULTS}

We have compared the recognition performance of Eigenfaces [4], Fisherfaces and SKKUfaces methods by using two different kinds of facial image database.

\subsection{Experiment I: Recognition Under Severe Illumina- tion Changes}

We use two image sets, SKKU database [8] and YALE database [9]. The size of a facial image is $50 \times 40$. The SKKU database consists of facial images captured in simple backgrounds, which are gathered under variations of luminance, facial expression, glasses, and time interval. The database contains 100 images of 10 persons. The YALE database is constructed in a similar fashion as the SKKU database. As shown in Table 1, the SKKUfaces method has lower error rate. We can see that the SKKUfaces method performs better than the others in the case of severe illumination changes. We have also found that the SKKUfaces method has computational advantages of reducing space and time complexity over the Fisherfaces method. The SKKUfaces method only need to compute matrices of size of $2000 \times 100$ and $2000 \times 10$ for within-class covariance and between-class covariance, respectively, while the other methods require computation of matrices of size $2000 \times 2000$.

\subsection{Experiment II: Recognition Under Normal Illumi- nation Changes}

Facial images of size $50 \times 50$ are obtained from eight people under lighting conditions (60 380lux), facial expression, pose (swing within $30^{\circ}$ degree). The total number of images is 230 images composed of 15 40 images per person. We have used 115 images for training and the others for testing. We have examined FRR (false reject Rate) and FAR (false accept rate). The error rates used for comparison is EER (equal error rate) that is the point where the values of FRR and FAR are equal. As shown in Table 1, the Fisherfaces and the SKKUfaces methods are better than the Eigenfaces method and the Fisherfaces method has lower ERR than the SKKUfaces method.

\subsection{Discussion}

The experimental results show that the SKKUfaces method is more appropriate to use than the Fisherfaces method in the case of severe illumination changes. We can see that face variations due to severe illumination changes are effectively removed sacrificing some information for the recognition of faces. In the normal illumination condition, the Fisherfaces method is the one that has to be used for face recognition.
Table 1 Error rates for the three methods (\%)

\begin{tabular}{|c|c|c|c|}
\hline \multirow{2}{*}{ Method } & \multicolumn{2}{|c|}{ Experiment I (FRR) } & \multirow{2}{*}{ Experiment II (EER) } \\
\cline { 2 - 4 } & SKKU DB & YALE DB & \\
\hline Eigenfaces & 45 & 30 & 9.4 \\
\hline Fisherfaces & 12 & 8 & 1.1 \\
\hline SKKUfaces & 9 & 4 & 2.0 \\
\hline
\end{tabular}

\section{CONCLUSIONS}

We have presented a real-time system for face detection and recognition for the application to access control to a building or an office. The system has been designed and implemented focusing on robustness to lighting variations and processing speed. The performance of the system will be enhanced with the development of a more intelligent method of switching the recognition modes according to the lighting condition.

\section{REFERENCES}

[1] Phillips, P.J., Hyeonjoon Moon, Rizvi, S.A., and Rauss, P.J., "The FERET evaluation methodology for face-recognition algorithms", IEEE Transactions on Pattern Analysis and Machine Intelligence, vol.22, pp. 1090-1104, 2000.

[2] R. S. Berns, Principles of Color Technology, John Wiley \& Sons Inc., pp. 22-23, 2000.

[3] P. Belhumeur, J. Hespanha, and D. Kriegman, "Eigenfaces vs. Fisherfaces: Recognition Using Class Specific Linear Projection", IEEE Trans. on PAMI, vol.19,no. 7, pp. 711- 720, 1997

[4] M. Turk and A. Pentland, "Eigenfaces for Recognition", Journal of Cognitive Neuroscience, vol. 3, no. 1, pp. 71-86, 1991

[5] M. Kirby and L. Sirovich, "Application of the KarhunenLoeve Procedure for the Characterization of Human Faces", IEEE Trans. on PAMI, vol .12, no .1, pp. 103-108, 1990.

[6] K. Etemad and R. Chellappa, "Discriminant Analysis for Recognition of Human faces image", Journal of Optical Society of America, vol. 14, no. 8, pp. 1724-1733, 1997.

[7] J. Yi, H. Yang and Y. Kim, "Enhanced Fisherfaces for Robust Face Recognition", Lecture Notes in Computer Science, Vol. 1811, pp. 50 2-511, 2000.

[8] http://vulcan.skku.ac.kr/research/skkufaces.html

[9] http://cvc.yale.edu/projects/yalefaces/yalefaces.html

[10] J. Cai and A. Goshtasby, "Detecting Human Faces in Color Images", International Workshop on Multi-Media Database Management Systems, pp. 124-131, 1998. 